(D)

CORPUS PUBLISHERS

\section{Corpus Journal of Dairy and} Veterinary Science (CJDVS)

\section{Volume 2 Issue 3, 2021}

Article Information

Received date : November 07, 2021

Published date: November 22, 2021

\section{*Corresponding author}

Dra Nancy Rodriguez, Molecular Biology Department, Faculty of Exact, Physical Chemical and Natural Sciences, Institute of Environmental Biotechnology and Health (INBIAS), National University of Rio Cuarto (UNRC)-National Council of Scientific and Technical Research (CONICET), National Route $36 \mathrm{KM}$ 601, X5804ZAB Rio Cuarto, Córdoba, Argentina

Keywords

PNIPAM co-APTA; Hydrogel Surface; Cell Adhesion/Release; Hydrogel-Pig Sperm Interactions; Sperm Selection

DOI: 10.54026/CJDVS1028

Distributed under Creative Commons CC-BY 4.0

\title{
Development of Poly-N- Isopropylacrylamide Surfaces for the Selection of Swine Sperm
}

Gricelda Morilla ${ }^{1}$, Ana Cecilia Liaudat ${ }^{1}$, Damián Blois $^{1}$, Virginia Capella ${ }^{1}$, Claudia Rivarola $^{2}$, Cesar Barbero ${ }^{2}$, Pablo Bosch ${ }^{1}$ and Nancy Rodríguez ${ }^{*}$

${ }^{1}$ Molecular Biology Department, Faculty of Exact, Physical Chemical and Natural Sciences, Institute of Environmental Biotechnology and Health (INBIAS), National University of Rio Cuarto (UNRC)-National Council of Scientific and Technical Research (CONICET), National Route 36 KM 601, X5804ZAB Rio Cuarto, Córdoba, Argentina

${ }^{2}$ Chemistry Department, Faculty of Exact, Physical-Chemical and Natural Sciences, Institute of Research in Energy Technologies and Advanced Materials (IITEMA), National University of Rio Cuarto (UNRC)-National Council of Scientific and Technical Research (CONICET), National Route 36 KM 601, X5804ZAB Rio Cuarto, Córdoba, Argentina

\section{Abstract}

The reproductive efficiency of pig farms is directly correlated with the fertility of the boars. The aim of this work was to develop polymeric materials that can be used as a platform to select a subpopulation of sperm with better cell physiological parameters. Polymeric hydrogels composed of Poly- $\mathrm{N}$-isopropylacrylamide with different positive charges given by copolymerization with (3-acrylamidopropyl) trimethylammonium chloride (APTA, 5-10-15\%), were synthesized. Subsequently, the interaction between the sperm cells and the polymeric surfaces was analyzed in TALP medium. Release of the spermatozoa from the polymeric surfaces was induced by changing to $\mathrm{Ca}^{2+}$ free media. Sperm motility, cell viability, plasma membrane and acrosome integrity were evaluated. The results indicated that a higher percentage of swine sperm attached to PNIPAM co-15\% APTA hydrogels $(62.86 \pm 3.33 \%)$. Ninety seven percent $(97.19 \pm 1.45 \%)$ of the sperm released from the PNIPAM co-15\%APTA surfaces were viable ( $<<0.05$ vs unbound population and raw semen), with acceptable motility $(58.89 \pm 1.28 \%)$ and with intact plasma and acrosomal membranes $(69 \pm 1.2 \%$ and $98.5 \pm 0.65 \%$ respectively). These results indicate that hydrogels can be used to select boar sperm with high viability and mobility for use in assisted reproductive techniques.

\section{Introduction}

Pig production is gaining great economic importance due to the greater demand for pork, as a result, at least in part, to an ncrease in exports worldwide (Gabozi, 2020). In most reproductive system of farm animals, different sperm selection techniques are used, such as swim-up, density gradient centrifugation, and passage through glass wool filters. These, focus on the selection of spermatozoa with better mobility and morphology [1], but do not report on possible genetic alterations o their ability to fertilize [1]. In this sense, there are other emerging sperm selection methods that are based on the integrity and maturity of the sperm membrane. In addition, selection techniques that are being developed have the ability to differentiate and include within the selection parameters of motility, viability, morphology, plasma membrane and acrosome integrity, production of reactive oxygen species and the ability to bind to the zona pellucida [2]. In this context, techniques that allow for the selection of the most suitable sperm, ideally analogous to the female reproductive system, would improve the results of assisted reproduction methods. Hydrogels are biomaterials made up of hydrophilic cross-linked polymeric structures capable of absorbing and retaining large amounts of water. This characteristic gives them an elastic, insoluble and soft consistency, similar to the extracellular matrix [3]. The structure and mechanical properties of the hydrogel vary depending on the monomer and copolymers used and the degree of crosslinking. In this way, a single monomer does not provide good water retention capacity and good mechanical characteristics at the same time, so it is advisable to resort to co-polymerization to achieve this result [4]. The most widely used thermosensitive hydrogel is Poly N-Isopropylacrylamide (PNIPAM), a material that has a phase transition temperature close to $32{ }^{\circ} \mathrm{C}$ in water (core body temperature of adult pigs: $39.2^{\circ} \mathrm{C}$ ). Studies carried out in our laboratory $[5,6]$ described the development of PNIPAM hydrogels copolymerized with other monomers such as 3-((acrylamidopropyl) trimethyl-ammonium chloride, APTA) in order to increase the temperature transition phase and bring it closer to swine body temperature [7]. In addition, it was shown that PNIPAM co-polymers cytotoxicity is very low, a feature that enhances their use with different biological samples $[5,6,8,9]$. Due to their low toxicity, high biocompatibility and the possibility of customizing their physical-chemical characteristics, such as functional groups and electrical charge, hydrogels are proposed as very attractive polymeric substrates to be applied in different biological fields, including the development of techniques for sperm selection. In this sense, recently published works by our laboratory describe the use of PNIPAM hydrogel surfaces copolymerized with $20 \%$ N-Tris (hydroxymethyl) methyl acrylamide and semi-interpenetrated with hyaluronic acid for the selection of bull sperm [10,11].

Based on the previous considerations, the aims of our work were the following: to develop new polymeric materials composed of PNIPAM copolymerized with APTA that serve as a binding substrate for swine sperm and to characterize the selected populations according to selected parameters of sperm quality. Results from our study indicated that the spermatozoa that attached and released from the surface of PNIPAM co-15\% APTA hydrogels possess appropriate values of motility, viability, plasma membrane integrity and acrosome integrity.

Materials and Methods

Synthesis of hydrogels 
The hydrogels were synthesized by radical copolymerization of $\mathrm{N}$-isopropylacrylamide monomers (NIPAM, Sigma Aldrich) copolymerized with the cationic monomer 3-(acrylamidopropyl) trimethyl-ammonium chloride (APTA) in different proportions with respect to PNIPAM (APTA 5\%, APTA $10 \%$, APTA 15\%) as described by Rivero et al. [6]. The crosslinking agent used was N-methylenebisacrylamide (BIS, Aldrich) and, as a water-soluble initiator, ammonium persulfate (APS, $1 \mathrm{mg} / \mathrm{mL}$, Aldrich). In addition, a catalyst, $\mathrm{N}, \mathrm{N}, \mathrm{N}^{\prime}, \mathrm{N}^{\prime}$ tetramethylenediamine (TEMED, $10 \mu \mathrm{L} / \mathrm{mL}$, Aldrich) was added. Finally, the $\mathrm{O}_{2}$ was removed by sparging with $\mathrm{N}_{2}$ gas. Upon completion of polymerization, the hydrogels were washed with distilled water three times a day, at room temperature for 5 days, in order to remove chemicals that have not reacted and may affect sperm viability.

\section{Semen samples}

The semen samples were provided by a swine artificial insemination center (CIP, Río Cuarto, Argentina). The analysis of the different parameters evaluated in this work was carried out with semen from different boars. The samples were preserved with commercial extender (Androstar plus, Minitube, Tiefenbach, Germany) and were kept at $15{ }^{\circ} \mathrm{C}$ until use. The samples were homogenized and centrifuged for 3 minutes at 300 $\mathrm{x} \mathrm{g}$ and resuspended in $1 \mathrm{ml}$ of sperm TALP medium [12] with the addition of bovine serum albumin $(6 \mathrm{mg} / \mathrm{ml}$, BSA, Sigma-Aldrich). For the different assays, an aliquot of boar spermatozoa ( $1 \times 10^{6}$ cells) was added to each well containing a hydrogel (PNIPAM co-5\% APTA, PNIPAM co-10\% APTA, or PNIPAM co-15\% APTA) and were incubated for $30 \mathrm{~min}$ at $37^{\circ} \mathrm{C}$ in TALP medium. Subsequently, the medium containing the unbound spermatozoa was removed. The percentage of sperm attached to the surface was obtained by the difference between the total numbers of sperms placed, less the number of sperm in the removed media [13]. Then, different hydrogels surfaces were washed three times with fresh medium and finally replaced by TALP medium without $\mathrm{CaCl}_{2}$ and $\mathrm{MgCl}_{2}$ for $30 \mathrm{~min}$ at $37^{\circ} \mathrm{C}$. The percentage of sperm released from each hydrogel was obtained by the difference between the attached sperm and the number of recovered sperm. The number of sperm released in both populations (unbound and released) was counted in a Neubauer chamber.

\section{Experimental design}

The experimental design is summarized in Figure 1. Briefly, sperm samples were washed with TALP medium [12] and added $\left(1 \times 10^{6} \mathrm{sperm} /\right.$ well $)$ to multi-well plates containing the different hydrogels (PNIPAM co-5\% APTA, PNIPAM co-10\% APTA or PNIPAM co$15 \%$ APTA). After $30 \mathrm{~min}$, unbound cells were removed and the percentage of adhered sperm was calculated. Sperm release was induced by incubating hydrogels in TALP medium without $\mathrm{CaCl}_{2}$ for $30 \mathrm{~min}$. Subsequently, the percentage of released sperm was calculated and biological characteristics such as sperm viability, motility, and acrosome and plasma membrane integrity were assessed.

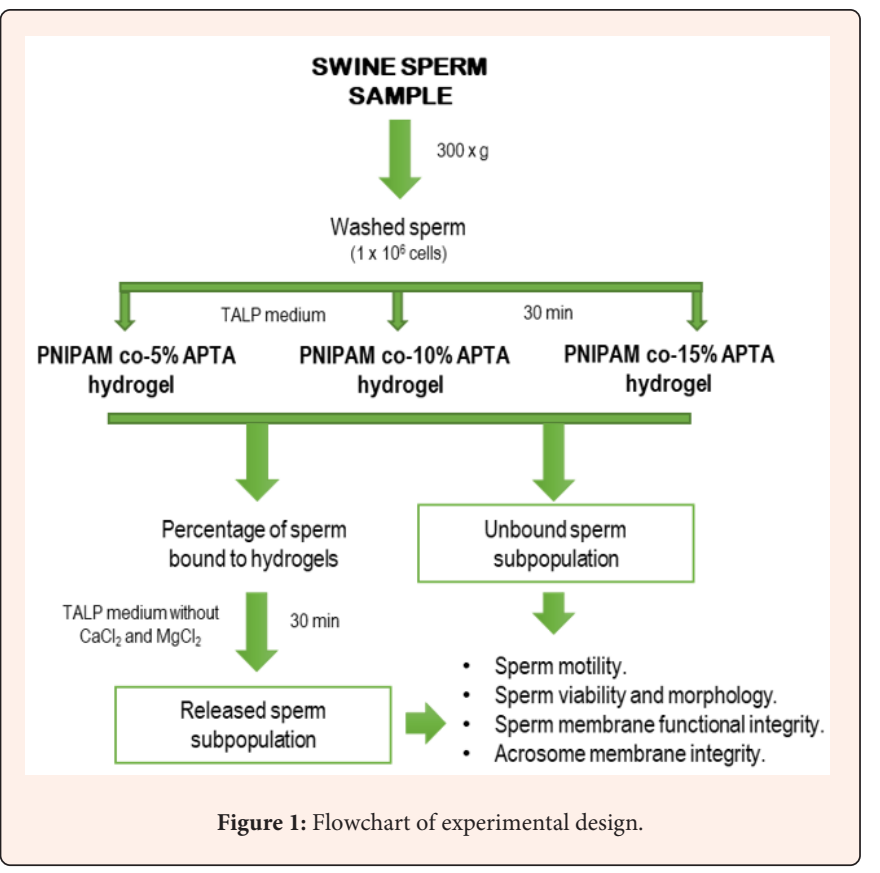

\section{Semen sample handling procedure}

An aliquot of sperm $\left(1 \times 10^{6}\right.$ cells / well $)$ was added to each well containing the hydrogel (PNIPAM co-5\% APTA, PNIPAM co-10\% APTA or PNIPAM co-15\% APTA) and subsequently incubated for $30 \mathrm{~min}$ at $37^{\circ} \mathrm{C}$ in TALP medium with $\mathrm{CaCl}_{2}$. The interaction between the sperm and the different hydrogels were examined under an inverted microscope. The TALP medium containing the unbound population was removed from the wells and the number of sperm not adhered to the different surfaces was calculated [13]. Then, the hydrogels were washed five times with medium and finally replaced by equal amount of TALP medium without $\mathrm{CaCl}_{2}$ and $\mathrm{MgCl}_{2}$ and incubated for $30 \mathrm{~min}$. The percentage of sperm released from each was calculated as described in semen sample section. Three intra-assay controls were included: a group of sperm was kept with TALP medium with $\mathrm{CaCl}_{2}$ throughout the selection process to evaluate the role of calcium in the sperm release process from the different surfaces. At the same time, the effect of the absence of $\mathrm{CaCl}_{2}$ in the entire sperm selection process was analyzed, a treatment that showed the importance of calcium in sperm attachment to surfaces. Finally, a group of spermatozoa were placed in TALP medium with $\mathrm{CaCl}_{2}$ without the presence of hydrogels in order to demonstrate the effect of the manipulations on sperm quality.

\section{Sperm motility}

The evaluation of sperm motility was carried out by phase contrast microscopy. A small drop of each treatment was placed on a slide tempered at $37^{\circ} \mathrm{C}$. A tempered coverslip was placed on the drop, and the motility of the spermatozoa was observed at $200 \mathrm{x}$ magnifications in an optical microscope, evaluating the total motile count. The percentage of total motility was subjectively evaluated by visual estimations of the same technician throughout the study (semen sample, washed sperm, unbound/released fraction). Sperm motility was determined by estimating the proportion of motile or immobile cells by counting at least 100 spermatozoa [13].

\section{Sperm viability}

Sperm viability was assessed by cell staining with propidium iodide (IP, Sigma). After sperm selection, $2 \times 10^{5}$ cells were suspended in $0.2 \mathrm{ml} \mathrm{PBS}$ with $5 \mu \mathrm{g} / \mathrm{ml} \mathrm{IP}$ and then incubated in the dark for 15 minutes at $37^{\circ} \mathrm{C}$. IP staining was analyzed by fluorescence microscopy with excitation and emission settings of 484 and $500 \mathrm{~nm}$, respectively. In order to be able to determine the total number of cells of the different populations studied, the spermatozoa were stained for 15 minutes with $1 \mu \mathrm{g} / \mathrm{ml}$ of Hoechst 33258 (Sigma). Subsequently, the samples were fixed with fixing solution (1 glycerol: 1 PBS) and analyzed by fluorescence microscopy. The acquisition of the image of the staining with Hoechst 33258 was carried out in the excitation range $360 \pm 40 \mathrm{~nm}$ and emission of $461 \pm 50 \mathrm{~nm}$. Images were obtained using a fluorescence microscope with a Nikon digital camera attached (Nikon Corporation, Konan, Minoto-Ku, Tokyo, Japan) and analyzed using Image J software (National Institute of Mental Health, Maryland, USA).

\section{Plasma membrane integrity}

The Hypoosmotic Swelling Test (HOST) evaluated the functionality of the sperm membrane. When cells were exposed to a hypoosmotic environment, live spermatozoa allow water to pass, causing cellular swelling or any degree of tail helical twisting ("swelling"). This effect is not observed in dead cells $[14,15]$. The hypoosmotic swelling test was adapted from Jeyendran et al. [16]. Briefly, $10 \mu \mathrm{L}$ of semen was incubated in $500 \mu \mathrm{L}$ of hypoosmotic solution $(0.45 \% \mathrm{NaCl})$ at $37{ }^{\circ} \mathrm{C}$ for $30 \mathrm{~min}$. The samples were examined using a phase contrast microscope. At least 200 sperm were counted per treatment. The percentage of cells showing rolled tails was considered to have an integral plasma membrane.

\section{Acrosome reaction}

Sperm from the different treatments were fixed in $1 \%$ glutaraldehyde, washed with $100 \mathrm{mM}$ ammonium acetate ( $\mathrm{pH}$ 9) and subsequently stained with $0.22 \%$ Coomassie brilliant blue for 2 minutes. The samples were examined by phase contrast microscopy and the photomicrographs were taken with a Nikon digital camera (Nikon Corporation, Konan, Minato-ku, Tokyo, Japan). Cells with blue staining in the apical area of the head were considered intact, while those with no staining in the same region were considered reacted.

\section{Statistical analysis}

Data were statistically analyzed by one-way analysis of variance (ANOVA) followed by the Bonferroni test, which was used as a post-hoc test. Infostat software was used to

Citation: : Morilla G, Liaudat AC, Blois D, Capella V, Rivarola C, et al. (2021) Development of Poly-N-Isopropylacrylamide Surfaces for the Selection of Swine Sperm. Corpus J Vet Dairy Sci 2: 1028 
analyze collected data [17]. Differences among groups were considered significant at $\mathrm{p}<0.05$.

\section{Results}

Swine sperm adhesion on PNIPAM co-APTA surfaces

Our results demonstrate that a subpopulation of viable swine sperm is able to adhere to PNIPAM hydrogels copolymerized with different percentages of APTA. In order to analyze if hydrogels surfaces synthesized with neutral amide polymers (monomeric unit: N-isopropylacrylamide, PNIPAM) with different concentrations of positive charge (APTA), have the ability to interact with boar sperm, cells attached/released capacity was obtained by exposing the sperm to different hydrogels surfaces. As shown in the images of Figure 2, swine sperm adhere to the different hydrogels surfaces. The quantification of the degree of binding showed that the percentage of sperm bound to the surfaces of PNIPAM co-15\% APTA was $62.86 \pm 3.33 \%$ and $49.26 \pm 3.33$ for PNIPAM co- $10 \%$ APTA A lower percentage of binding was observed on the surfaces of PNIPAM co-APTA $5 \%(25.34 \pm 3.33 \%, \mathrm{p} \leq 0.05)$. The percentage of sperm released from PNIPAM co-15\% APTA hydrogels was significantly larger than that calculated for PNIPAM co-5\% APTA hydrogel ( $\mathrm{p} \leq 0.01$, Figure 2$)$.

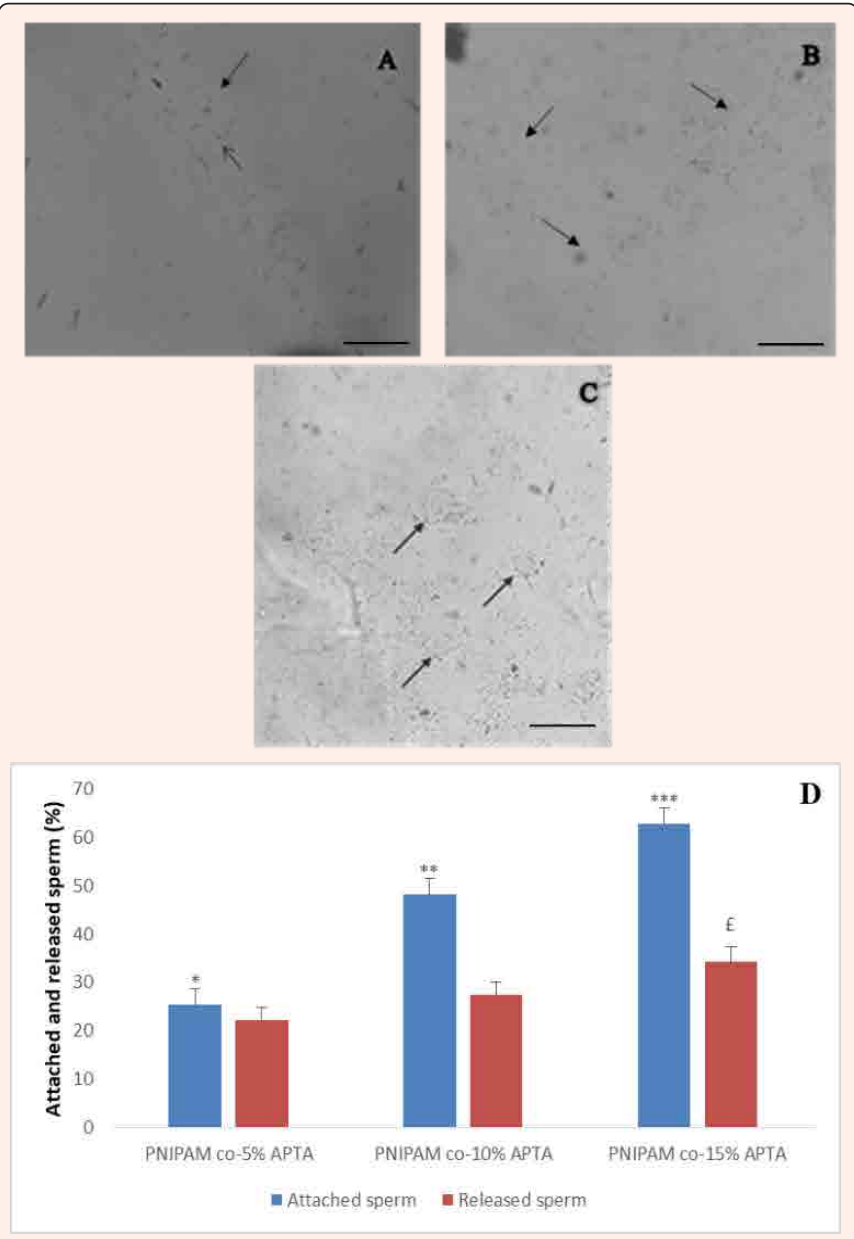

Figure 2: Phase contrast microscopy of swine sperm attached to PNIPAM co-5\% APTA (A), PNIPAM co-10\% APTA (B) and PNIPAM co-15\% APTA (C) surfaces. The arrows indicate sperm attached to the different hydrogels. Bar scale: $250 \mu \mathrm{m}$. D: Graph of boar sperm adhesion and release after exposure to the different hydrogels (PNIPAM co-APTA 5\%, PNIPAM co-10\% APTA, PNIPAM co-15\%-APTA). Values represent means \pm SEM. $n=6 .{ }^{*} \mathrm{p}<0.05$ vs adhered PNIPAM co- $10 \%$ APTA and PNIPAM co- $15 \%$ APTA, ${ }^{* *} \mathrm{p}<0.05$ vs adhered PNIPAM co- $5 \%$ APTA and PNIPAM co- $15 \%$ APTA, ${ }^{* * *} \mathrm{p}<0.05$ vs adhered PNIPAM co-5\% APTA and PNIPAM co- $10 \%$ APTA, $£ \mathrm{p}<0.05$ vs released PNIPAM co- $5 \%$ APTA
As to mass motility, $67.78 \pm 1.28 \%$ of the spermatozoa attached to the PNIPAM co$15 \%$ APTA surfaces presented very good to good motility and $58.89 \pm 1.28 \%$ of the released sperm showed good total motility. As shown in Figure 3, 2.81 \pm 1.45 of cells that were released from PNIPAM co-15\% APTA surfaces were non-viable, a result significantly lower than the percentage found in unbound cells, in the raw sperm and washed sperm $(\mathrm{p}<0.05)$. As can be seen in Figure 4, the sperm released from the PNIPAM-co-15\% APTA hydrogels present $67.73 \pm 4 \%$ of the plasma membranes with deformities. A difference is observed between the other populations selected from the surfaces of PNIPAM co-5\% APTA and PNIPAM co-10\% APTA. When evaluating the integrity of the acrosome membrane, $95.35 \pm 1.18 \%$ of the spermatozoa not bound to the PNIPAM co-15\% APTA surface, showed an intact membrane. In the released sperm, this evaluated parameter was $98.5 \pm 0.65 \%$

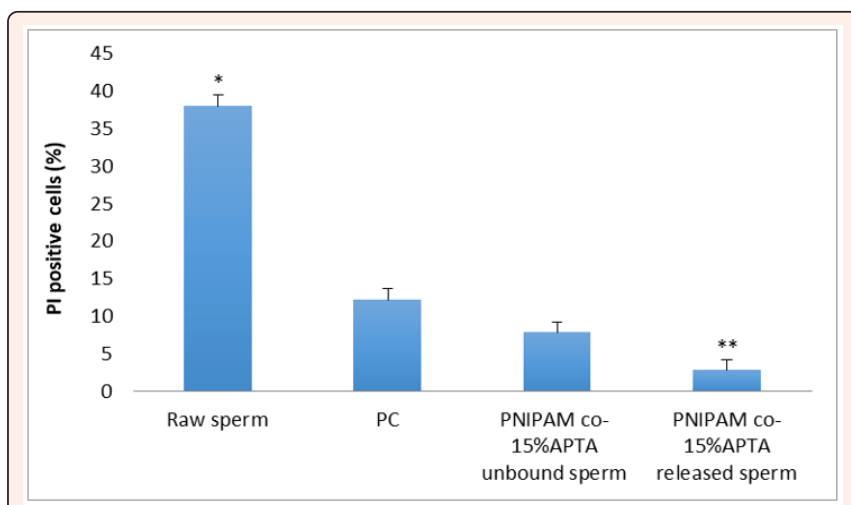

Figure 3: Viability of unbound and released swine sperm after exposure to PNIPAM co-15\% APTA surfaces, in the initial raw semen sample and after sperm washing process (WS). ${ }^{*} \mathrm{p}<0.05$ vs PC, unbound PNIPAM co-15\% APTA and released PNIPAM co- $15 \%$ APTA. ${ }^{* *} \mathrm{p}<0.05$ vs PNIPAM co- $15 \%$ APTA unbound. Values are expressed as means \pm SEM of at least three independent replicates.

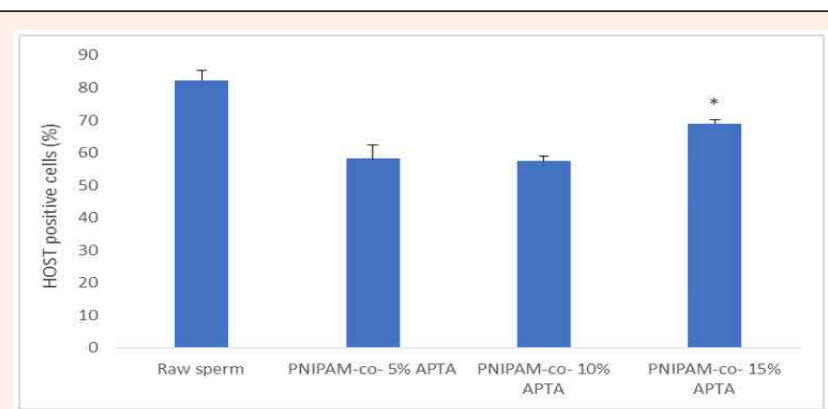

Figure 4: Percentage of HOST positive cells (with deformation of the plasma membrane) of raw sperm and in released from the different hydrogels samples (PNIPAM-co-5\% APTA, PNIPAM-co-10\% APTA, PNIPAM-co-15\% APTA). Values represent the means \pm SEM of at least three independently performed experiments. ${ }^{*} \mathrm{p}<0.05$ vs PNIPAM co- $10 \%$ APTA, PNIPAM co- $5 \%$ APTA and raw sperm.

\section{Discussion}

The aim of this work was to analyze PNIPAM hydrogels copolymerized with different percentage of 3- (acrylamidopropyl) trimethyl-ammonium chloride for the selection of swine spermatozoa. Our results demonstrated that a subpopulation of boar spermatozoa binds to PNIPAM co-APTA surfaces and the cells were released under $\mathrm{Ca}^{2+}$ free conditions. The released sperm showed acceptable values of motility, viability, plasma membrane integrity, and low incidence of acrosome reaction. As with the different sperm selection methods, the goal is to obtain the best sperm to be used in ART. In most sperm selection techniques, centrifugation is one of the most used steps for semen procedures. Some examples of the most used selection methods are the density gradient and the swimup [18]. Different studies show that the manipulation and centrifugation of male gametes generates an increase in the production of Reactive Oxygen Species (ROS). ROS induce peroxidative damage of sperm plasma membrane, leading to decrease in sperm viability,

Citation: : Morilla G, Liaudat AC, Blois D, Capella V, Rivarola C, et al. (2021) Development of Poly-N-Isopropylacrylamide Surfaces for the Selection of Swine Sperm. Corpus J Vet Dairy Sci 2: 1028 
motility and fertilizing capacity (Aitken and Clarkson, 1988). In addition, ROS could cause DNA damage, a factor implicated as a causative agent of decreased fertility, early abortions, and increased risk of transmitting undesirable genetic defects to the offsprings (Ozmen, et al. 2007). Since high levels of ROS are the main cause of infertility in males, it is important to avoid them or reduce their production (Homa et al. 2015). According to Li et al. [18], centrifugation time and speed are crucial in this regard. In this work, short centrifugation times ( $2 \mathrm{~min}$.) and low revolutions/min (3000 RPM) were used, in order to reduce the alterations produced by ROS as much as possible.

During the maturation process, the sperm incorporates surface glycoproteins that gives it a net negative charge and facilitates the interaction of sperm cells with the extracellular medium, preventing them from self-agglutinating or binding to inadequate sites of the female reproductive tract [1]. Sperm attachment to the oviduct occurs by direct contact, through ligand-receptor interaction between lectins present in the apical area of the sperm and specific carbohydrate moieties on the plasma membrane of oviductal cells. This binding is species-specific and calcium-dependent [19]. Additionally, it has been described that there are several adhesion proteins called cadherins, presen in both sperm and oviduct cells. These intervene in the formation of the oviductal reservoir and it is a process in which calcium is decisive [20]. When we evaluated the sperm adhesion on the PNIPAM co-APTA hydrogels $(5,10,15 \%)$ it was observed that the sperm bound heterogeneously to the surfaces and that the highest percentage of binding was obtained with PNIPAM co-15\% (62.86 $\pm 3.33 \%)$. The TALP manipulation medium [12] contains $\mathrm{HCO}^{3-}, \mathrm{CaCl}_{2}$ and albumin among its components, which favor the stability of the spermatozoa in the handling manipulation environment. These elements of the environment are essential for sperm maturation and capacitation in most species [21]. Sperm maturation and capacitation are physiological processes that normally occur in the female reproductive tract and are necessary to acquire the ability to fertilize the oocyte [22]. In these experiments we were able to show that the increase in the percentage of APTA and the presence of $\mathrm{CaCl}_{2}$ in the handling medium favor the union of boar sperm. When the manipulation medium is replaced by TALP without $\mathrm{CaCl}_{2}$, the release of cells attached to the different surfaces is stimulated. The use of different types of hydrogels is in high development and are being used as support for different cell lines (stem cells, fibroblasts, cells of kidney origin) as well as for the regeneration of cartilage or cardiac lesions [23]. Furthermore, in different studies performed in our laboratory it was shown that hydrogels have high biocompatibility $[5,8,9]$ and with bull sperm $[10,11]$. Hydrogels could be physically and chemically modified, feature that gives another advantage to the system (Vega 2002, Benítez et al. 2015)

Sperm motility is an indicator of cell viability and is directly related to membrane integrity and intracellular metabolism. The control and regulation of sperm movement is a complex system, where are substances that positively regulate this parameter, including extracellular calcium [14]. Furthermore, this cation is essential in the acrosomal formation and reaction process. Studies carried out by Holt et al. [24] in swine and Amann et al. [25] in cattle, showed that the type of motility is suggestive of the quality of the ejaculate. Studies carried out in other species, for example, [26] in rabbits and Moore and Akhondi [27] in rats, indicate a direct relationship between motility and fertility. Sperm cell motility assessment is one of the most widely used methods, due to its low cost, speed and simplicity. On the other hand, O'Connor et al. [28], compared computerized motility assessment techniques with conventional tests in bovine species, and indicated that there is not such a significant advantage of computerized analyzes over traditional sperm motility techniques. In our study, a high percentage of motility was observed in the initial sample, a parameter that is conserved when sperm bind to the surfaces of hydrogels. The decrease in motility after sperm selection is probably due to manipulation during this selection process. In sperm viability analysis by propidium iodide staining, a technique that is based on the exclusion of the dye by cells that have the plasma membrane intact (living cells) [29,30], it was observed that the cells released from the PNIPAM co-15\% APTA surfaces showed a higher percentage of viability compared to the unbound cells. This was the case with both the initial sample (fresh semen) and with the sample after the centrifugation process. Studies by Holý [31], indicate that a semen sample is considered good if it has less than $30 \%$ dead sperm.

Using the Hypo-Osmotic Swelling Technique (HOST), the functional capacity of the plasma membrane is assessed, taking into account its osmotic properties and the reesterification capacity of non-specific cell esterases. It is known that a membrane that is structurally damaged is not functional, but surely not all structurally intact membranes are necessarily functional [14,32]. It is important that this parameter be preserved as it is a sine qua non condition of all vital cells processes [33]. In addition, the membrane integrity allows the selective transport of fluids and molecules [34]. In our results, we observed a high percentage of deformation of the sperm membrane of the sperm released from the different hydrogels: PNIPAm co-5\% APTA (58.2 $\pm 4.3 \%$ ), PNIPAM co- $10 \%$ APTA $(57.4 \pm 1.4 \%)$, PNIPAM co-15\% APTA $(69 \pm 1.2 \%)$. These values suggest that the released sperm have their plasma membrane functional. Den Daas [35] demonstrated that, if damage occurs to the sperm plasma membrane, this damage is irreversible, so the intact cell membrane is essential to indicate that a sperm is viable. The acrosome is located at the apical end of the sperm head and contains different hydrolytic enzymes such as hyaluronidase, acrosine, zone glycine, esterase, phospholipase A, acid phosphatase, histamine, protamine, etc., which are important in the acrosome reaction since they are responsible for the penetration of the zona pellucida as part of fertilization process. The determination of acrosome status is a predictive test that evaluates sperm damage and, therefore, the fertility potential of the semen sample [36]. Coomassie blue staining is a simple and reliable technique for determining acrosome status [37]. In an ideal sample, the percentage value with damaged acrosome membrane should be less than $15 \%$ [38]. In our experience, by monitoring the presence of reacted sperm throughout the selection process, we were able to find a high percentage of unreacted sperm (as shown in the results section). The $95.35 \pm 1.18 \%$ of the spermatozoa not bound to the PNIPAM co$15 \%$ APTA surface showed an intact membrane, and, in the released spermatozoa, this evaluated parameter was $98.5 \pm 0.65 \%[39,40]$. The results indicate that sperm selected by PNIPAM co-15\%APTA surfaces are suitable for use in assisted reproduction techniques.

\section{Conclusion}

Here, we report for the first time the use of PNIPAM copolymerized APTA hydrogels for swine sperm selection. Boar sperm was able to attach to PNIPAM co-APTA surfaces and a significant percentage of spermatozoa were released from PNIPAM co$15 \%$ APTA surfaces. Select sperm have acceptable values of motility, viability, sperm membrane integrity and a high percentage of sperm with intact acrosomal membrane.

\section{Acknowledgement}

Gricelda Morilla and Ana Cecilia Liaudat have contributed equally to this study. Pablo Bosch and Nancy Rodríguez are both senior authors.

\section{References}

1. Sánchez Pozo MCS, Prieto IS, Rodríguez GB (2017) Advanced techniques for sperm selection. Journal of the Clinical Laboratory 10(3): 129-138.

2. Morrell JM, Rodriguez Martinez H (2010) Practical applications of sperm selection techniques as a tool for improving reproductive efficiency. Vet Med Int 14(3): 572580 .

3. Mullarney MP, Seery TAP, Weiss RA (2006) Drug diffusion in hydrophobically modified N, N-dimethylacrylamide hydrogels. Polymer 47: 3845-3855.

4. Tibbitt MW, Anseth KS (2009) Hydrogels as imitations of the extracellular matrix for 3D cell culture. Biotechnol Bioeng 103(4): 655-663.

5. Rivero R, Alustiza F, Capella V, Liaudat C, Rodríguez N, et al. (2017) Physicochemical properties of ionic and non-ionic biocompatible hydrogels in water and cell culture conditions: Relation with type of morphologies of bovine fetal fibroblasts in contact with the surfaces. Colloids and Surfaces B: Biointerfaces 158: 488-497.

6. Rivero RE, Alustiza F, Rodríguez N, Bosch P, Miras MC, et al. (2015) Effect of functional groups on physicochemical and mechanical behavior of biocompatible macroporous hydrogels. Reactive and Functional Polymers 97: 77-85.

7. Vihola H, Laukkanen A, Valtola L, Tenhu H, Hirvonen J (2005) Cytotoxicity of thermosensitive polymers poly ( $\mathrm{N}$-isopropylacrylamide), poly (N-vinylcaprolactam) and amphiphilically modified poly (N-vinylcaprolactam). Biomaterials 26(16): 3055-3064.

8. Rivero R, Capella V, Liaudat C, Bosch P, Barbero C, et al. (2020) Mechanical and physicochemical behavior of a 3D hydrogel scaffold during cell growth and proliferation. RSC Advances 10(10): 5827-5837.

9. Capella V, Rivero R, Liaudat C, Ibarra L, Roma D, et al. (2019) Cytotoxicity and bioadhesive properties of poly-N-isopropylacrylamide hydrogel. Heliyon 5(4): 1474 .

10. Liaudat AC, Blois D, Capella V, Morilla G, Rivero R, et al. (2021) Bull sperm selection by attachment to hyaluronic acid semi-interpenetrated hydrogels. Reproduction in Domestic Animals.

11. Blois D, Liaudat C, Capella V, Morilla G, Rivero R, et al. (2021) Interaction between hyaluronic acid semi-interpenetraded hydrogel with bull spermatozoa: studies of sperm attachment-release and sperm quality. Advance Marterals.

12. Parrish JJ, Susko Parrish JL, Winer MA, First NL (1988) Capacitation of bovine sperm by heparin. Biol Reprod 38(5): 1171-1180.

Citation: : Morilla G, Liaudat AC, Blois D, Capella V, Rivarola C, et al. (2021) Development of Poly-N-Isopropylacrylamide Surfaces for the Selection of Swine Sperm. Corpus J Vet Dairy Sci 2: 1028 
13. Bosch P, de Avila JM, Ellington JE, Wright RW (2001) Heparin and $\mathrm{Ca}^{2+}$-free medium can enhance release of bull sperm attached to oviductal epithelial cell monolayers. Theriogenology 56(2): 247-260.

14. Gadea J, Matás C, Lucas X (1998) Prediction of porcine semen fertility by homologous in vitro penetration (hIVP) assay. Anim Reprod Sci 54(2): 95-108.

15. Rubio Guillén JL, Quintero Moreno AA, González Villalobos DM (2009) Effect of cryopreservation on the integrity of the plasma and acrosomal membrane of bull spermatozoa. Scientific Magazine 19(4): 382-389.

16. Jeyendran RS, Van Der Ver HH, Perez Pelaez M, Crabo BG, Zaneveld LJ (1984) Development of an assay to assess the functional integrity of the human sperm membrane and its relationship to other semen characteristics. J Reprod Fertil 70(1): 219-228.

17. Di Renzo JL, Casanoves F, Balzarini MG, Gonzalez L, Tablada M, et al. (2018) Grupo InfoStat, FCA, Universidad Nacional de Córdoba. Argentina.

18. Li Z, Zhou Y, Liu R, Lin H, Liu W, et al. (2012) Effects of semen processing on the generation of reactive oxygen species and mitochondrial membrane potential of human spermatozoa. Andrologia 44(3):157-163.

19. Suarez S, Revah I, Lo M, Kölle S (1998) Bull sperm binding to oviductal epithelium is mediated by a $\mathrm{Ca} 2+$ dependent lectin on sperm that recognizes Lewis-a trisaccharide. Biol Reprod 59(1): 39-44

20. Veiga MF (2011) Studies on the presence and expression of epithelial cadherin in human and murine gametes. Evidence on their participation in the fertilization process. University of Buenos Aires, Faculty of Exact and Natural Sciences, Argentina.

21. Visconti P, Galantino Homer H, Moore G, Bailey J, Ning X, et al. (1998) The molecular basis of sperm capacitation. J Androl 19(2): 242-248.

22. Arnoult C, Kazam IG, Visconti PE (1999) Control of the low voltage-activated calcium channel of mouse sperm by egg ZP3 and by membrane hyperpolarization during capacitation. Proc Natl Acad Sci USA 96(12): 6757-6762.

23. Burdick JA, Prestwich GD (2011) Hyaluronic acid hydrogels for biomedical applications. Adv Mater 23(12): H41-H56.

24. Holt C, Holt WV, Moore HD, Reed HC, Curnock RM (1997) Objectively measured boar sperm motility parameters correlate with the outcomes of on farm inseminations results of two fertility trials. Journal of Andrology 18(3): 312-323.

25. Amann RP (1989) Can the fertility potential of a seminal sample be predicted accurately? J Androl 10(2): 89-98.

26. Farrell PB, Foote RH, Simkin ME, Clegg ED, Wall RJ (1993) Relationship of semen quality, number of sperm inseminated, and fertility in rabbits. J Androl 14(6): 464 471.

27. Moore HD, Akhondi MA (1996) Fertilizing capacity of rat spermatozoa is correlated with decline in straight-line velocity measured by continuous computer- aided sperm analysis: epididymal rat spermatozoa from the proximal cauda have a greater fertilizing capacity in vitro than those from the distalcauda or vas deferens. J Androl 17(1): 50-60.

28. Connor MT, Amann RF, Saacke RG (1981) Comparisons of computer evaluations of spermatozoal motility with standard laboratory tests and their use for predicting fertility. J Anim Sci 53: 1368-1376.

29. Brito LFC, Barth AD, Bilodeau Goeseels S, Panich PL, Kastelic JP (2003) Comparison of methods to evaluate the plasmalemma of bovine sperm and their relationship with in vitro fertilization rate. Theriogenology 60(8): 1539-1551.

30. Yániz JL, Palacín I, Vicente Fiel S, Gosalvez J, López Fernández C, et al. (2013) Comparison of membrane-permeant fluorescent probes for sperm viability assessment in the ram. Reprod Domest Anim 48(4): 598-603.

31. Holý L (1987) Biology of bovine reproduction: Laboratory examination of the fertility of the stallion bull. Cuba, pp. 367-381.

32. Zubair M, Ahmad M, Jamil H (2015) Review on the screening of semen by hypoosmotic swelling test. Andrologia 47(7): 744-750.

33. Correa JR, Zavos PM (1994) The hypoosmotic swelling test: Its employment as an assay to evaluate the functional integrity of the frozen-thawed bovine sperm membrane. Theriogenology 42(2): 351-360.

34. Avery S, Bolton VN, Mason BA (1990) An evaluation of the hypo-osmotic sperm swelling test as a predictor of fertilizing capacity in vitro. Int J Androl 13(2): 93-99.

35. Den Daas N (1992) Laboratory assessment of semen characteristics. Anim Reprod Sci 28(1-4): 87-94

36. Cross NL, Meizel S (1989) Methods for evaluating the acrosomal status of mammalian sperm. Biology of reproduction 41(4): 635-641.

37. Larson JL, Miller DJ (1999) Simple histochemical stain for acrosomes on sperm from several species. Mol Reprod Dev 52(4): 445-449.

38. Angel MO, Echeverri CAG (2014) Reproductive tissue culture and production and manipulation of bovine embryos.

39. Duarte C, Núñez V, Wong Y, Vivar C, Benites E, et al. (2017) Impact of the Z potential technique on reducing the sperm DNA fragmentation index, fertilization rate and embryo development. JBRA Assist Reprod 21: 351-355.

40. Llóveras M (2013) Greater artificial insemination in pigs. Field Supplement, La Nación, Argentina, pp. 07-20.

41. Memon A, Wahid H, Rosmina Y, Ebrahimi M, Nadia F (2012) Effect of antioxidants on post thaw microscopic, oxidative stress parameter and fertility of boer goat spermatozoa in Tris egg yolk glycerol extender. Animal Reproduction Science 136(1-2): 55-60.

42. Ordóñez Arias WW (2017) Efficacy of ultraviolet radiation in the sterilization of porcine seminal plasma and its behavior on sperm quality (Master's thesis).

43. Torreta ME (2007) Artificial insemination with refrigerated semen in pigs. 